\title{
First Case of MELAS Syndrome Presenting with Local Brain Edema Requiring Decompressive Craniectomy
}

\author{
Osman YESILBAS ${ }^{1}$, Esma SENGENC ${ }^{2}$, Melike ERSOY OLBAK ${ }^{3}$, Derya BAKO ${ }^{4}$, Oznur Gokce NIZAM ${ }^{5}$, \\ Mehmet Hakan SEYITHANOGLU ${ }^{6}$, Davut PEHLIVAN ${ }^{7}$, Serdar CEYLANER ${ }^{8}$, Dilara ICAGASIOGLU², \\ Kursad AYDIN ${ }^{9}$
}

\begin{abstract}
${ }^{1}$ Karadeniz Technical University, Faculty of Medicine, Department of Pediatrics, Division of Pediatric Critical Care Medicine, Trabzon, Turkey ${ }^{2}$ Bezmialem Vakif University, Faculty of Medicine, Department of Pediatrics, Division of Pediatric Neurology, Istanbul, Turkey ${ }^{3}$ Health Sciences University, Bakirkoy Dr. Sadi Konuk Research and Education Hospital, Department of Pediatrics, Division of Pediatric Metabolism, Istanbul, Turkey

${ }^{4}$ Health Sciences University, Van Training and Research Hospital, Departments of Radiology, Division of Pediatric Radiology, Van, Turkey

${ }^{5}$ Bezmialem Vakif University, Faculty of Medicine, Department of Pediatrics, Istanbul, Turkey

${ }^{6}$ Bezmialem Vakif University, Faculty of Medicine, Department of Neurosurgery, Istanbul, Turkey

${ }^{7}$ Baylor College of Medicine, Department of Pediatrics, Division of Pediatric Neurology and Developmental Neuroscience, Houston, USA

${ }^{8}$ Intergen Genetics Center, Division of Clinical Genetics, Ankara, Turkey

${ }^{9}$ Medipol University, Faculty of Medicine, Department of Pediatrics, Division of Pediatric Neurology, Istanbul, Turkey
\end{abstract}

Corresponding author: Osman YESILBAS drosmanyesilbas@gmail.com

\section{ABSTRACT}

Mitochondrial encephalomyopathy, lactic acidosis, and recurrent stroke-like episodes (MELAS) syndrome is a rare but one of the most common maternally inherited multisystem disorder. Although patients with MELAS present a variable clinical profile, strokelike lesions have been detected in $90 \%$ of cases, with stroke being the first presenting symptom in $25 \%$ of cases. However, cases of local brain edema requiring decompressive craniectomy has not been reported.

A 12-year-old male patient was admitted to our pediatric intensive care unit with altered mental status, seizures, and vision loss. The patient was stuporous and presented neck stiffness. Complete blood count, serum electrolytes, biochemistry (including lactate level), acute phase reactants, and repeated blood gas analysis were unremarkable. Brain magnetic resonance imaging (MRI) revealed an edematous stroke-like lesion in the right occipital lobe accompanied by brain swelling. Intravenous ceftriaxone, acyclovir, intravenous immunoglobulin (IVIG), and pulse steroid therapy were started for possible diagnosis of viral/bacterial/autoimmune encephalitis; levetiracetam, phenytoin, and an infusion of sodium thiopental were started for refractory status epilepticus; and a $3 \% \mathrm{NaCl}$ infusion was started for local brain edema. The results of serum autoimmune encephalitis panel were negative. Further investigations for rheumatic, vascular, and metabolic disorders were unremarkable. Despite these supportive treatments, the patient was clinically decompensated due to brain swelling that progressed to the left midline shift, and he underwent decompressive craniectomy. Histologic examination of brain biopsy specimen revealed non-specific encephalitis findings. A pathogenic variant of the MT-TL1 gene (m.3243A>T), responsible for MELAS, was detected. The patient's condition dramatically improved after specific treatment for MELAS.

If the diagnosis and treatment are delayed, MELAS syndrome can cause serious brain edema, which may ultimately require decompressive craniectomy.

KEYWORDS: Child, Brain edema, Decompressive craniectomy, MELAS syndrome

ABBREVIATIONS: MELAS: Mitochondrial encephalomyopathy, lactic acidosis, and stroke-like episodes, MRI: Magnetic resonance imaging, DWI: Diffusion-weighted imaging, PICU: Pediatric intensive care unit, CSF: Cerebrospinal fluid, EEG: Electroencephalogram, HSV: Herpes simplex virus, PCR: Polymerase chain reaction, ADC: Apparent diffusion coefficient

\begin{tabular}{|c|c|c|c|c|}
\hline Osman YESILBAS & (1) : 0000-0002-4290-0491 & Oznur Gokce NIZAM & (D) : 0000-0003-2615-3307 Dilara ICAGASIOGLU & (iD) : 0000-0002-7728-2611 \\
\hline Melike ERSOY OLBAK & (1) : 0000-0002-2316-0790 & Davut PEHLIVAN & (1) : 0000-0001-5788-0270 & \\
\hline Derya BAKO & (D) : 0000-0003-0642-6793 & Serdar CEYLANER & (D) : 0000-0003-2786-1911 & \\
\hline
\end{tabular}




\section{- INTRODUCTION}

$\mathrm{M}$ itochondrial encephalomyopathy, lactic acidosis, and stroke-like episode (MELAS) syndrome is one of the most frequent maternally inherited mitochondrial diseases. It is a multi-organ disorder with broad manifestations, including stroke-like episodes, seizures, lactic acidemia, recurrent headaches, myopathy, cortical vision loss, hemiparesis, hearing impairment, recurrent vomiting, altered consciousness, diabetes, developmental delay, peripheral neuropathy, learning disabilities, memory impairment, myoclonus, ataxia, psychotic disorders, cardiomyopathy, cardiac conduction abnormalities, and nephropathy. Most affected individuals develop symptoms of MELAS between the ages of two and 40 years $(2,13)$. On conventional magnetic resonance imaging (MRI) and diffusion-weighted imaging (DWI), stroke-like lesions are identified as essential imaging features of MELAS. These lesions predominantly affect the posterior lobes of the brain and are not confined to the vascular territories (12).

In this article, we report an extremely rare case of MELAS syndrome that required urgent decompressive craniectomy for severe brain edema. According to the available English literature, this is the first reported case in which decompressive craniectomy was performed to ameliorate MELAS syndromerelated severe brain edema. Written informed consent was obtained from the patient's parents.

\section{CASE REPORT}

A 12-year-old male patient was referred to our pediatric intensive care unit (PICU) with altered mental status, seizures, and vision loss. The patient's medical history revealed that approximately 2 years ago, he had been admitted to another hospital with fever, altered mental status, and seizures. He had been treated for seizures and meningoencephalitis based on physical and cerebrospinal fluid (CSF) examinations and brain MRI results (Figure 1A, B). His electroencephalogram $(E E G)$ findings were unremarkable. Results of polymerase chain reaction (PCR) assay for herpes simplex virus (HSV), CSF culture, and serum autoimmune encephalitis panel were negative. He was discharged from the hospital with a prescription of oral levetiracetam after completion of antibiotic and antiviral treatment. For approximately two years since discharge, he had been experiencing loss of consciousness with accompanying visual symptoms lasting 15-20 minutes. During this period, results of a repeat EEG, serum autoimmune encephalitis panel, acyl-carnitine profile, serum lactate, plasma amino acids, and urine organic acid levels were unremarkable. Since significant regression was observed on the brain MRI (Figure 1C), the condition had been attributed to encephalitis sequelae, and he was treated with levetiracetam for epilepsy.

When he was referred to our institution in a comatose stage (Glasgow Coma Scale score of 9), his pupils were isochoric, and pupillary light reflex was present in both eyes. Blood pressure, heart/respiratory rate, and body temperature were all within the normal ranges for his age. Respiratory effort was sufficient, lung auscultation was normal, and pulse oxygen saturation in room air was $96-98 \%$. Results of complete blood count with differential, serum electrolytes, biochemistry (including lactate level), acute phase reactants, and blood gas analysis were unremarkable. MRI revealed midline, subuncal, and central herniation due to the mass effect of stroke-like lesions involving the right parietal, temporal, and occipital lobes (Figure 1D). Magnetic resonance angiography and venography scans were unremarkable. Lumbar puncture was deferred because of herniation risk. The background rhythm in the patient's electroencephalogram was irregular and slow. Mechanical ventilatory support was initiated after the rapid sequence intubation protocol. Intravenous (IV) ceftriaxone, acyclovir, IV immunoglobulin (IVIG, $2 \mathrm{~g} / \mathrm{kg}$ over 5 days), and pulse steroid therapy ( $30 \mathrm{mg} / \mathrm{kg}$ for 5 days) were started for possible diagnosis of viral/bacterial/autoimmune encephalitis; levetiracetam, phenytoin, and an infusion of sodium thiopental were started for refractory status epilepticus; a $3 \% \mathrm{NaCl}$ infusion was started for local brain edema (serum sodium level was targeted between 160 and $165 \mathrm{meq} / \mathrm{L}$ ); subcutaneous enoxaparin was started to treat the stroke-like lesions found on the brain MRI, and fentanyl infusion was started for analgesia. Results of serum autoimmune encephalitis panel, plasma acyl-carnitine profile, serum lactate, plasma amino acids, urine organic acids, and further investigations for rheumatic and vascular diseases were unremarkable.

On the fifth day of PICU admission, anisocoria, bradycardia, and hypertension emerged when his serum sodium level was $163 \mathrm{meq} / \mathrm{L}$. Intravenous mannitol $(4 \times 0.5 \mathrm{gr} / \mathrm{kg})$ treatment was promptly added to therapies and a repeat MRI (Figure 1E) was performed immediately. Because of the progression of brain herniation, decompressive hemicraniectomy was performed. After decompressive hemicraniectomy, the herniation gradually regressed (Figure 1F). Histologic examination of brain biopsy specimen revealed non-specific encephalitis findings.

A pathogenic mutation $m .3243 A>T$ was detected in the MTTL1 gene, and MELAS was diagnosed on the eighth day of PICU hospitalization. A high $(10 \mathrm{~g} / \mathrm{m} 2)$ IV dose of L-arginine, coenzyme Q10 (10 mg/kg per day), B vitamins cocktail, and levocarnitine $(100 \mathrm{mg} / \mathrm{kg} /$ day) were promptly administered for the treatment of MELAS. He was extubated, and non-invasive mechanical ventilatory support was initiated on the end of the third day since the MELAS-specific therapies were initiated.

The patient's condition gradually improved over the course of his hospitalization and he was discharged without any respiratory support on the thirty-sixth day of admission. At discharge, he could understand and speak fluently, but his vision was impaired. Subsequently, he was followed by a pediatric metabolism and neurology specialist, with oral arginine, coenzyme Q10, L-carnitine, vitamin B cocktail, and levetiracetam. During a two-year follow-up, he started to walk with support, but unfortunately, he still could not see clearly.

\section{DISCUSSION}

Although the underlying pathophysiology of MELAS syndrome is not fully understood, impaired mitochondrial energy production, microvasculature angiopathy, and nitric oxide 


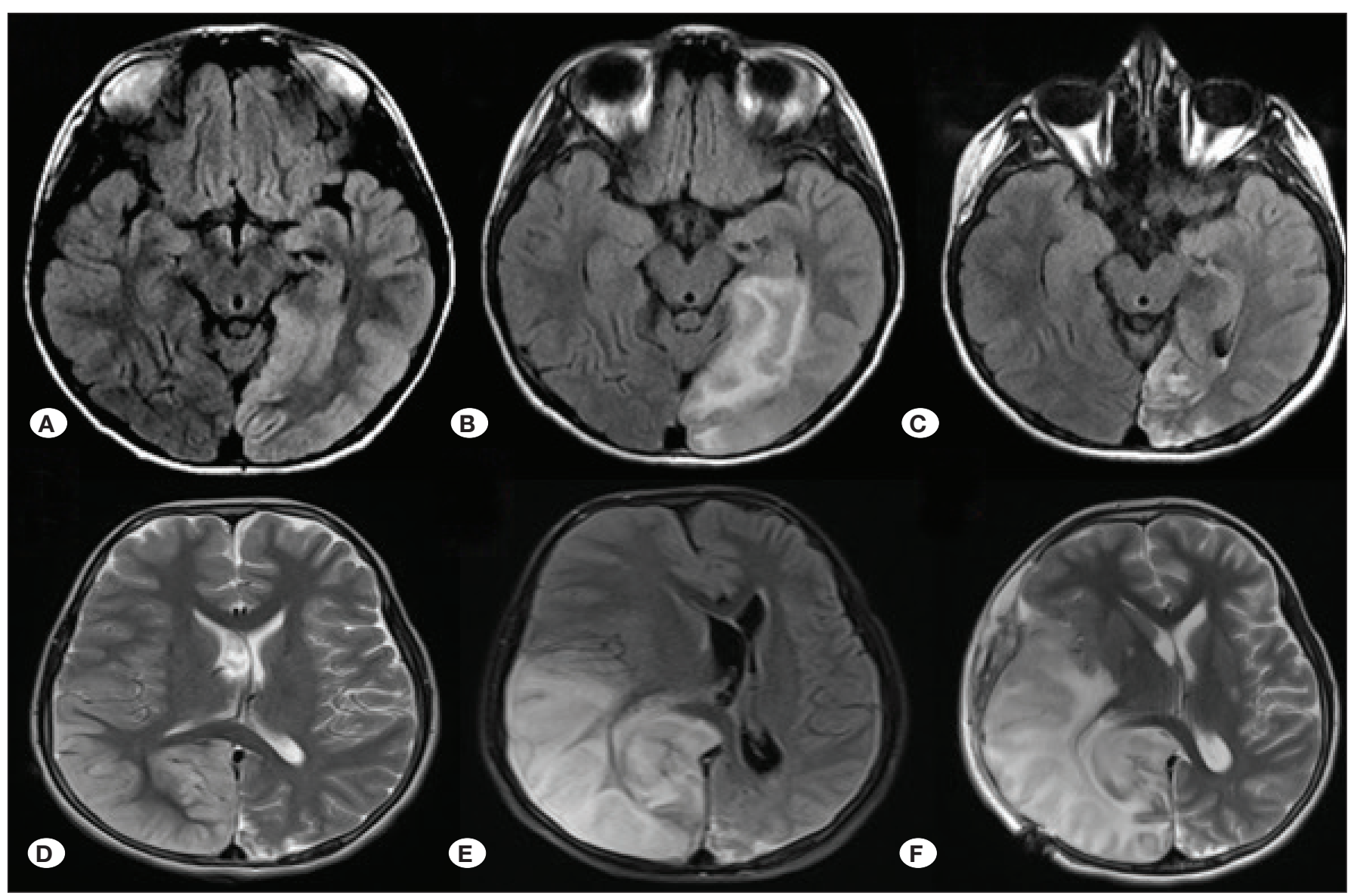

Figure 1: First row (A-C): First hospitalization of the patient in another hospital. A) Axial FLAIR magnetic resonance imaging (MRI) performed on the first day of admission shows mild swelling involving the cortex and subcortical areas and hyperintensity in the left temporo-occipital lobe. B) Progression of in cortical and deep subcortical swelling with deep white matter involvement on the thirteenth day. C) One month later (after hospital discharge): slight hyperintensity and atrophy in the left temporo-occipital lobe is noted, indicative of previous stroke-like lesions. Second row: Second hospitalization of the patient in our pediatric intensive care unit. D) Axial T2weighted MRI was performed on the day of admission. Mild swelling involving the cortex and subcortical area and hyperintensity in the right parieto-occipital lobe is seen. E) Axial FLAIR MRI was performed on the fifth day of admission. A midline shift is seen due to the increasing mass effect of the stroke-like lesion involving the right parietal, temporal, and occipital lobes. Involvement of the cingulate gyrus is seen, causing subfalcine herniation. F) T2-weighted MRI was performed after decompressive craniectomy. Slightly regressed midline shift is noted-a finding indicative of the mass effect of a stroke-like lesion and persistence of subfalcine-midline herniation.

deficiency are possible interacting mechanisms secondary to various mutations, especially in the MT-TL1 gene, similar to that in our patient (2). The m.3243A $>\mathrm{G}$ pathogenic variant in the mitochondrial gene MT-TL1 causes symptoms in approximately $80 \%$ of individuals. Stroke-like episodes, which occur in $84-99 \%$ of affected individuals, are the main manifestation of MELAS $(2,3)$. These episodes clinically emerge with partially reversible aphasia, headaches, altered mental status, seizures, cortical vision loss, and motor weakness secondary to progressive neurological impairment, as in our patient $(2,11)$. MELAS syndrome usually present clinically in adolescents and adults. Most patients are between 5 and 15 years of age similar to our patient (15). The diagnosis of MELAS syndrome is challenging because the clinical manifestations, which may be related to the functioning of many organ systems, are often non-specific. At the first hospitalization, our patient was misdiagnosed with encephalitis because of his clinical manifestations (fever, neck stiffness, neurological dysfunction, and seizures) and biochemical features of CSF. Zhang et al. (15) reported a case of 13-year-old male patient who was misdiagnosed with encephalitis, similar to our patient. In our patient, the loss of consciousness with accompanying visual symptoms for approximately two years was attributed to epileptic attacks secondary to encephalitis sequela because his brain MRI results improved during this period. Although lactic acidosis, especially elevated lactate levels in the CSF, are essential signs of this syndrome, such levels can be normal in a minority of individuals with MELAS syndrome (9). In our patient, no increase in serum lactate was observed during hospitalization. Since lumbar puncture was not performed in our PICU because of possible herniation risk, CSF lactate levels could not be investigated. 
Occasionally, conventional neuroimaging methods may fail to distinguish this disease from ischemic stroke and meningoencephalitis $(6,15)$. Classical T2-weighted brain $\mathrm{MRI}$ findings include increased signal intensity abnormalities that simulate ischemic stroke predominate in the occipital, parietal, and temporal lobes in patients with MELAS. These brain lesions can be unilateral or bilateral, frequently involving the cortex and mostly affecting the gray matter more than white matter. The affected areas and their borders do not correspond to a specific vascular distribution. Brain magnetic resonance angiography usually yields no findings of abnormalities $(2,6,7,8,15)$. The lesions are not necessarily permanent and may sometimes show contrast enhancement due to the increased permeability resulting from reperfusion and congestion secondary to blood-brain barrier damage. Clinical and radiological improvements may be observed with time $(5,8)$. Initially, our patient was misdiagnosed with meningoencephalitis, but his clinical and radiological findings improved later, which is compatible with the data reported in the literature. A characteristic hallmark of MELAS, which was also seen in our patient, is that recurrence may occur in different locations with each attack (5).

DWI mostly shows an increased apparent diffusion coefficient (ADC) in stroke-like lesions of MELAS, in contrast to the decreased ADC seen in ischemic strokes $(5,8)$. This feature of MELAS strongly suggests that vasogenic, but not cytotoxic, edema is a predominant characteristic in the stroke-like lesion of this syndrome. Breakdown and increased permeability of the blood-brain barrier, secondary to mitochondrial respiratory failure in the endothelial cells and smooth muscles of the cerebral arterioles, are speculated to be the underlying pathophysiology $(1,14)$. Although there are a few reported cases of brain edema secondary to MELAS syndrome in the literature $(10,14)$, to the best of our knowledge, our patient is the first patient in whom decompressive craniectomy was performed for severe brain edema.

Magnetic resonance spectroscopy (MRS) is another ancillary non-invasive method for diagnosing stroke-like episodes in MELAS. These episodes are associated with elevated lactate peaks and low $\mathrm{N}$-acetylaspartate/choline ratios. Additionally, serial MRS imaging can be used to monitor clinical and radiological improvement after MELAS-specific treatment, such as IV L-arginine administration (4).

\section{CONCLUSION}

Diagnosis of MELAS syndrome is challenging because the clinical manifestations are often non-specific. Important radiological findings for the diagnosis of stroke-like episodes include brain MRI findings of lesions that predominantly affect the posterior lobes not confined to the vascular territories. Although lactic acidosis, especially elevated lactate levels in the CSF, are essential signs of this syndrome; however, lactate levels can be normal in a minority of individuals. Clinicians should be aware that if the diagnosis and treatment are delayed, MELAS syndrome can cause serious brain edema, requiring decompressive craniectomy.

\section{REFERENCES}

1. Breningstall GN, Lockman LA: Massive focal brain swelling as a feature of MELAS. Pediatr Neurol 4:366-370, 1988

2. El-Hattab AW, Adesina AM, Jones J, Scaglia F: MELAS syndrome: Clinical manifestations, pathogenesis, and treatment options. Mol Genet Metab 116:4-12, 2015

3. Hirano M, Pavlakis SG: Mitochondrial myopathy, encephalopathy, lactic acidosis, and stroke like episodes (MELAS): Current concepts. J Child Neurol 9:4-13, 1994

4. Hovsepian DA, Galati A, Chong RA, Mazumder R, DeGiorgio CM, Mishra S, Yim C: MELAS: Monitoring treatment with magnetic resonance spectroscopy. Acta Neurol Scand 139: 82-85, 2019

5. Khandwala K, Ahmed A, Sheikh T: MELAS: A complex and challenging diagnosis. J Coll Physicians Surg Pak 28:46-48, 2018

6. Kolb SJ, Costello F, Lee AG, White M, Wong S, Schwartz ED, Messé SR, Ellenbogen J, Kasner SE, Galetta SL: Distinguishing ischemic stroke from the stroke-like lesions of MELAS using apparent diffusion coefficient mapping. J Neurol Sci 216:1115, 2003

7. Lorenzoni PJ, Werneck LC, Kay CS, Silvado CE, Scola $\mathrm{RH}$ : When should MELAS (Mitochondrial myopathy, Encephalopathy, Lactic Acidosis, and Stroke-like episodes) be the diagnosis? Arq Neuropsiquiatr 73:959-967, 2015

8. Majoie CB, Akkerman EM, Blank C, Barth PG, Poll-The BT, den Heeten GJ: Mitochondrial encephalomyopathy: Comparison of conventional MR imaging with diffusion-weighted and diffusion tensor imaging: Case report. AJNR Am J Neuroradiol 23:813-816, 2002

9. Matsumoto J, Saver JL, Brennan KC, Ringman JM: Mitochondrial encephalomyopathy with lactic acidosis and stroke (MELAS). Rev Neurol Dis 2:30-34, 2005

10. Purucker M, Schröder R, Harms L, Timm G, Zierz S, Ludolph AC: Reversible focal brain edema in a patient with MELAS (mitochondrial myopathy, encephalopathy, lactic acidosis, and stroke-like episodes) syndrome. J Epilepsy 9:79-82, 1996

11. Sproule DM, Kaufmann P: Mitochondrial encephalopathy, lactic acidosis, and stroke like episodes: Basic concepts, clinical phenotype, and therapeutic management of MELAS syndrome. Ann N Y Acad Sci 1142:133-158, 2008

12. Xu W, Wen J, Sun C, Cao J, Li Y, Geng D: Conventional and diffusional magnetic resonance imaging features of mitochondrial encephalomyopathy, lactic acidosis, and stroke-like episodes in chinese patients: A study of 40 cases. J Comput Assist Tomogr 42:510-516, 2018

13. Yatsuga S, Povalko N, Nishioka J, Katayama K, Kakimoto N, Matsuishi T, Kakuma T, Koga Y; Taro Matsuoka for MELAS Study Group in Japan: MELAS: A nationwide prospective cohort study of 96 patients in Japan. Biochim Biophys Acta 1820:619-624, 2012

14. Yoneda M, Maeda M, Kimura H, Fujii A, Katayama K, Kuriyama M: Vasogenic edema on MELAS: A serial study with diffusionweighted MR imaging. Neurology 53:2182-2184, 1999

15. Zhang L, Zhou J: Differential diagnosis of MELAS syndrome from viral encephalitis by MRI: A case report. Radiol Infect Disea 5: 138-142, 2018 